\title{
Protée
}

\section{L’incipit esquivé}

\section{Jean-Pierre Vidal}

Volume 28, numéro 1, 2000

\section{Variations sur l'origine}

URI : https://id.erudit.org/iderudit/030582ar

DOI : https://doi.org/10.7202/030582ar

Aller au sommaire du numéro

Éditeur(s)

Département des arts et lettres - Université du Québec à Chicoutimi

ISSN

0300-3523 (imprimé)

1708-2307 (numérique)

Découvrir la revue

\section{Citer cet article}

Vidal, J.-P. (2000). L'incipit esquivé. Protée, 28(1), 37-44.

https://doi.org/10.7202/030582ar

\section{Résumé de l'article}

Si tous les commencements humains sont rétrospectifs et émergent comme des projections largement fantasmatiques, encore que logiques, à partir d'un présent arbitrairement immobilisé, nulle part mieux que dans un incipit de roman ce paradoxe temporel ne peut s'observer comme le véritable vortex de production sémiotique qu'il constitue. C'est ainsi qu'après avoir brièvement revisité le célèbre incipit du Voyage au bout de la nuit de Céline tel qu'en lui-même enfin celui du Voyeur de Robbe-Grillet le change, le présent article s'attache à montrer comment le personnage peut naître littéralement du paysage incipital en prenant pour exemple Le Neveu de Rameau de Diderot.
Ce document est protégé par la loi sur le droit d'auteur. L’utilisation des services d’Érudit (y compris la reproduction) est assujettie à sa politique d'utilisation que vous pouvez consulter en ligne.

https://apropos.erudit.org/fr/usagers/politique-dutilisation/ 


\section{L'IN CIPIT ESQ U IVÉ}

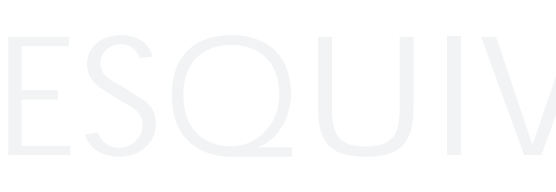

JEAN - PIERRE VIDAL

Le plus simple serait de ne pas commencer. Mais je suis obligé de commencer. C'est-à-dire que je suis obligé de continuer.

Beckett (L'Innommable)

Puisque aucun commencement ne nous est jamais strictement contemporain, puisque à la conscience tout est déport, puisqu'en elle tout début se trouve logiquement précédé par ce dont il est le début et qui n'a de lieu que d'un incommensurable présent, ce qui se marque expressément comme un commencement n'est jamais qu'une rétrospection plus ou moins lucide. Une analepse en quelque sorte mais une analepse qui serait paradoxalement première dans la mesure où c'est elle qui "ouvre», dans tous les sens du terme, y compris son acception heideggerienne. Tout commencement est ainsi une décision et une coupure. Tout commencement dit ainsi en même temps la voix qui l'énonce et qui rien qu'à sa façon de scander, de s'immobiliser un temps dans ce qui l'occupe, proclame qu'ici, mais où?, cela, mais quoi?, commence, ou plutôt, «a commencé».

Tout commencement déclaré, dans quelque champ ou discours que ce soit, est aussi un roman, plus ou moins familial dans sa volonté d'auto-fondation, un roman qui se met en branle sous nos yeux, une fiction en train de se donner lieu. C'est pourquoi peut-être rien n'éclaire mieux les commencements humains que le lieu de l'incipit romanesque.

Si le mot, en latin médiéval, apposé au début d'un manuscrit, avant même le titre de l'œuvre, désignait à l'origine un espace d'écriture équivalent à de simples guillemets ou à un rideau qui s'ouvre et se bornait donc, en fait d'effet de sens, à son déchiffrement immédiat par la lecture, à une ponctualité sitôt posée que dépassée, le lieu textuel qu'il désigne aujourd'hui est un incommensurable puisqu'il peut, selon les lectures et les structures qu'elles identifient comme faisant partie de la rhétorique du commencement, se limiter à la première phrase ou couvrir pratiquement tout un chapitre; on connait même un roman fait tout entier d'incipit, Si par une nuit d'hiver un voyageur d'Italo Calvino. 
En matière de roman proprement dit, tout incipit reprend implicitement - et c'est en grande partie l'effet, au niveau sémiotique, de la non-coïncidence, constitutive du lieu du sujet, entre la conscience et son objet, que j'évoquais en ouverture - l'indépassable formulation du Voyage au bout de la nuit: "Ça a débuté comme ça ${ }^{1}$. La répétition du déictique «ça» rend visible une boucle où le retour du même mot fait apparaître comme une symétrie ce qui, de fait, est une non-coïncidence puisque le premier "ça» renvoie à une indécision - quoi «ça»? - dont le deuxième se donne aussitôt comme la future résolution en forme d'index. Ainsi l'incipit se dédouble-t-il: au commencement était le «ça» d'un présent dont on ne sait encore rien sinon d'entrée (parce qu'il est ce qu'il est, c'est-à-dire une situation dont la voix du narrateur suffit à nous assurer qu'elle perdure et qu'elle est un aboutissement) que le «ça» d'un futur immédiat va venir lui expliciter un passé. Nous sommes donc dans un temps labyrinthique: comme pour le parcours de Thésée, le futur qu'est ici le récit c'est le passé des "événements", réels ou imaginaires, peu importe à cet égard leur statut, la seule chose qui compte à leur sujet, c'est qu'ils représentent le présent indépassable du dire. Et que ce présent-là va venir occuper tout l'espace du présent de la prise de parole initiale où le narrateur assurait son quant-à-soi comme un retrait. Le présent du dire engouffre le présent originaire du diseur dans la distance infime et capitale d'un "comme» où se dit, métaphorique et conditionnel, l'espace littéraire comme décision, fracture, irruption. Et capture du lecteur à l'«être-là» duquel se donnent par le fait même un nouvel espace et un nouveau temps. On sait comment Diderot, dans Jacques le fataliste, met expressément en scène cet espace paradoxal du dire occupé par les deux "voix», adverses et complices, du lecteur et de l'auteur, imaginaires tous deux, ou plutôt abstraits. On sait aussi comment Calvino ouvre son roman, du moins tendanciellement, sur la coïncidence du lecteur abstrait et du lecteur réel, tous deux écrasés dans l'instance de celui qui va entreprendre la lecture du dernier roman de Calvino, Si par une nuit d'hiver un voyageur, avant de les dissocier d'une façon d'autant plus spectaculaire qu'elle est progressive. Le texte en effet commence par faire des suggestions au lecteur avant de lui imaginer un lieu de lecture dont la description de plus en plus précise départagera définitivement le lieu de lecture de la fiction (qu'elle fait ainsi émerger de la confusion initiale des espaces du texte) du lieu de lecture, indescriptible par définition, de chacun des lecteurs «réels» du roman. Mais remarquons ici, quant à nous, la postérité que Robbe-Grillet, grand lecteur de Céline on le sait, a donnée, avec l'incipit du Voyeur à cette extraordinaire ouverture du Voyage.

Et pour bien voir la variation à l'œuvre, reportonsnous à la toute fin du roman de Céline:

De loin, le remorqueur a sifflé; son appel a passé le pont, encore une arche, une autre, l'écluse, un autre pont, loin, plus loin [...] Il appelait vers lui toutes les péniches $d u$ fleuve, toutes, et la ville entière, et le ciel et la campagne, et nous, tout qu'il emmenait, la Seine aussi, tout, qu'on n'en parle plus. ${ }^{2}$

Cette accumulation finale en guise de congé n'est pas sans évoquer la phrase finale de Dans le labyrinthe:

[...] mais la vue se brouille à vouloir en préciser les contours, de même que pour le dessin trop fin qui orne le papier des murs, et les limites trop incertaines des chemins luisants tracés dans la poussière par les chaussons de feutre, et, après la porte de la chambre, le vestibule obscur où la canne-parapluie est appuyée obliquement contre le porte-manteau, puis, la porte d'entrée une fois franchie, la succession des longs corridors, l'escalier en spirale, la porte de l'immenble avec sa marche de pierre, et toute la ville derrière moi. ${ }^{3}$

On voit ici à l'œuvre la même échappée spatiale (antérograde chez Céline, rétrograde chez RobbeGrillet, du moins par rapport à la position du narrateur), parcourue progressivement, comme si le roman, tout roman, ne pouvait se clore que sur une «somme», à valeur de résumé accélérateur (la fin de Madame Bovary, par exemple) ou cumulatif (ici), et ce, qu'il s'agisse d'un enfermement (Robbe-Grillet) ou d'une ouverture (Céline). On notera en tout cas dans les deux instances la dimension universalisante ("et la 
ville entière [...] tout» chez Céline, «et toute la ville derrière moi » chez Robbe-Grillet) qui montre bien qu'à son échelle, bien sûr, tout récit est une apocalypse, le dévoilement de la fin coïncidant avec une sorte de rassemblement d'objets, de lieux, de personnages, un peu comme cette parade finale que Fellini impose à ses personnages à la fin de Huit et demi. Et c'est, en dernière instance, toujours la fin qui fonde le début, ne serait-ce que cette fin provisoire qu'implique toute rétrospection par l'immobilisation imposée à un présent où l'on fait le point: « Ça a débuté comme ça» encore.

Si la séquence finale du roman de Céline propose une sorte d'illustration du «ça» initial comme objet réduit à un espace urbain que le récit liquide enfin, ou plutôt qu'il s'exhorte 4 à liquider («[...] qu'on n'en parle plus»), celle du roman de Robbe-Grillet montre, dans l'emboîtement d'une symétrie inverse, le progressif effacement de «tout» ce qui n'était pas le lieu initial, lequel, on le sait, est d'abord réduit à l'instance la plus minimale qui soit, celle d'un «être-là-maintenant» ("Je suis seul ici, maintenant, bien à l'abri») qui progressivement se scinde, se multiplie, s'aliène, à partir du développement d'une implication logique («seul» produisant "pas seul/l'autre/les autres», «ici» produisant "ailleurs», «maintenant» impliquant «autrefois» et «bien à l'abri» à la fois l'idée d'espace clos et de protection/menace qui va produire le personnage du «soldat», d'abord menaçant puis menacé.

Manifestement, ouverture et clôture vont de pair et si tout début «attend" toujours expressément sa fin, toute fin est aussi la relecture, la relance du début, comme si tout pouvait repartir pour un tour. Car toute fin, je l'ai dit, est toujours d'une certaine façon antérieure à son début. Peut-être d'ailleurs est-ce tout simplement parce qu'on ne peut jamais identifier quelque début que ce soit qu'à partir de la fin de ce qu'il initiait. Et que ce qui s'initiait là ne peut, justement, être perçu comme tel que lorsque son parcours est clos. Aussi n'est-ce pas seulement un secret de fabrication ou une recette, mais presque une ontologie littéraire que dévoile Edgar Poe, quand il recommande de toujours commencer... par la fin.
Mais venons-en enfin au Voyeur. Ce qui d'emblée paraîtra, une fois ces divers détours pris, plus troublant, c'est la façon dont son incipit semble présenter... la suite de la fin du Voyage. Qu'on en juge:

C'était comme si personne n'avait entendu. La sirène émit un second sifflement, aigu et prolongé, suivi de trois coups rapides, d'une violence à crever les tympans - violence sans objet, qui demeura sans résultat. ${ }^{5}$

Le remorqueur de Céline est ici un traversier et son appel demeure expressément lettre morte, mais il s'adresse dans un cas comme dans l'autre à la même universalité («L'une contre l'autre, toutes les têtes étaient dressées dans une attitude identique» lira-t-on six lignes plus bas dans le texte de Robbe-Grillet, en forme d'explicitation du "personne» initial). Et si l'appel chez Céline est une invite à prendre le large (y compris du récit qui ainsi s'achève), il est chez RobbeGrillet, comme il se doit, le signe, au contraire, que l'on touche terre et que l'île qu'est le récit, le cadre de la fiction étant ainsi la représentation du lieu de la lecture, sera aussi le moment d'une mémoire, réelle ou imaginaire: l'équivalent du «Ça a commencé comme ça» de Céline peut en effet fort bien se lire sous la forme du «On lui avait souvent raconté cette histoire» que l'on retrouve un peu plus bas dans la même page initiale du Voyeur, surtout si l'on prend soin de remarquer la même insistance déictique («cette histoire»).

On pourrait sans doute, si l'on s'en donnait un peu la peine, relever, dans l'histoire de la littérature romanesque, de nombreux incipit déployés comme des "suites", coup de chapeau plus ou moins conscient aux ancêtres qu'on se reconnaît et «dans» l'œuvre desquels on écrit toujours. Et peut-être l'attitude de Calvino, organisant des incipit de romans différents de façon qu'ils forment une suite (dont rend compte la phrase que forment leurs titres si on les met bout à bout), estelle le résultat ironique d'une prise de conscience aiguë de l'intertextualité en tant qu'elle est toujours foncièrement le moment où une lecture devient écriture.

Mais outre la référence à Céline, ce qui devrait particulièrement retenir l'attention dans cet incipit du 
Voyeur, c'est ce que j'appellerais une fiction virtuellement antécédentée; certes le roman moderne connaît bien cette façon d'introduire la lecture à une séquence temporelle en cours ${ }^{6}$ et sans doute l'incipit de Madame Bovary ("Nous étions à l'étude...») en est-il l'exemple le plus illustre, mais Robbe-Grillet donne une réalité plus prégnante encore à ce monde où brusquement, lecteurs, nous entrons, en imprimant à sa première phrase les contours sibyllins, tout de sousentendus, d'une formulation où la dimension déictique repose sur l'implicite et le quasi-instantané d'une présence narratrice se parlant en quelque sorte à elle-même, sans avoir besoin de nommer ce qu' "elle" vient d'entendre. Ainsi le lecteur se trouve-t-il d'entrée de jeu prisonnier d'un temps et d'un espace qui lui sont comme antérieurs et se proclament indépendants de lui, au point de lui «taire» quelque chose.

C'est sans doute chez Diderot que l'on retrouve les incipit où se marque le plus spectaculairement cette distance entre la lecture et la fiction qu'on lui tend et qu'elle produit, distance fondatrice, en fin de compte, de l'espace texte, ouvert toujours par cette noncoïncidence soudain théâtralement dévoilée, comme un impossible commencement. Voyons-y.

\section{LA FAMILIARITÉ DÉCONCERTANTE}

En ce XVIIIe siècle qui multiplie les interrogations sur les origines (de l'entendement humain, des connaissances humaines, des langues, des inégalités parmi les hommes, etc.), la Révolution française viendra dire qu'il ne s'agit jamais en fin de compte que de refonder l'ordre social en mettant fin à l'ordre ancien. Et si l'Encyclopédie travaille dans le faire-voir comme émergence délibérée d'un savoir devenant immédiatement politique, parce que d'entrée de jeu en attente d'un usage, Diderot met au contraire un soin particulier, dans ses œuvres romanesques, à nier l'événement du commencement. Il n'y va pas, bien sûr, sans quelque paradoxe puisque tout récit met en scène des événements, c'est-à-dire de la nouveauté donnée comme telle, quand bien même il s'agirait d'un leurre. Mais le souci explicite qu'il met à travailler dans l'intertexte exhibé (Les Mille et une Nuits, Don
Quichotte, Tristram Shandy, Rabelais, Ésope, etc.), ainsi que diverses stratégies textuelles, dont la moins étonnante n'est pas celle qui ouvre La Religieuse, font de Diderot le maître de l'incipit esquivé: «La réponse de M. le marquis de Croismare, s'il m'en fait une, me fournira les premières lignes de ce récit» 7 . Merveille de double entente et plaisante private joke ${ }^{8}$, cette ouverture, où derrière la narratrice se fait manifestement entendre la voix de Diderot, dispose le biographique dans la fiction, sensiblement comme le feront plus tard et Le Neveu de Rameau et Jacques le fataliste, en montrant déjà cette indécidabilité du lieu d'énonciation où l'amnésique post-modernité veut nous faire croire que, sous le nom d' "autofiction", quelques-uns de ses traits prétendument spécifiques s'identifient. Qui plus est, le «je» encore anonyme qui ouvre le récit et dont l'ambiguïté est grosse d'une scission progressive, comme si, j’y reviendrai, la narratrice ne devait se détacher qu'insensiblement sur fond de collectif et de générique ${ }^{9}$, ce «je» s'inscrit d'emblée dans ce que j'ai appelé, à propos du Voyeur, une «fiction virtuellement antécédentée». Et il attend une réponse pour y trouver les premières lignes de son récit, comme si ce qui vient de se lire n'était pas déjà le récit, ce que révélera d'ailleurs la suite puisque le récit des malheurs de la religieuse commence à ce récit du moment où elle a envoyé une lettre en forme d'appel au secours. Est-ce beaucoup forcer le texte dans le sens d'une modernité, au demeurant partout annoncée chez Diderot, que de voir dans cet incipit la représentation de l'appel au lecteur, sa présence avérée servant d'amorce à la participation que l'on attend de lui, à sa réponse, qui seule permettra à la fiction de se construire? Le passage, biographique, des lettres apocryphes "réelles" aux lettres imaginaires "prêtées" à un personnage dit bien, en tout cas, ce que représente pour l'auteur ce passage à l'œuvre en forme de passage à l'acte. En attente de ce qui sera son début (!), le roman s'ouvre sur une sorte d'aveu, mais encore voilé d'être à usage presque exclusivement intime, d'un méfait qui lui est à la fois extérieur et premier.

L'effet le plus marqué de cette stratégie incipitale c'est, me semble-t-il, d'ouvrir l'espace romanesque à 
une dialectique du sujet, chacun des deux protagonistes (appelons-les, par commodité, auteur et lecteur) s'y trouvant expressément, à sa place même et selon sa logique ou même son ontologie propre, déporté vers une indécision qui pourrait bien être la marque la plus claire de toute fiction. Car si tout texte est certes la rencontre sur le terrain des signes de deux consciences, cette rencontre n'est possible que de leur perte, au moins symbolique, d'identité dans le vaste et incertain creuset de la langue dont la part active qu'elle prend dans le processus a continuellement été sous-estimée par toutes les théories de la réception et leur absolutisation, très politiquement correcte, $\mathrm{du}$ pouvoir du lecteur.

Il reste cependant que si, dans l'espace anonyme du texte, Diderot «est» la religieuse au moins autant que Flaubert Madame Bovary, la réponse que la narratrice attend de M. de Croismare dit aussi celle espérée du lecteur non seulement pour qu'il prête vie au récit mais même, tout simplement, pour que le récit advienne ${ }^{10}$. Tout comme la familiarité, à la pérennité indéfinie, que Jacques entretient avec son maître, assoit la familiarité à conquérir entre le texte et sa lecture. Le «ils se sont rencontrés» de la fiction représente en effet le «nous nous rencontrons» de l'immédiat lecteur. Et aucun texte ne dit mieux que Jacques le fataliste le jeu de pertes, d'oublis et de substitutions sur lequel constamment se gage cette dernière rencontre. Il dit aussi, c'est connu, que la rencontre n'est jamais l'événement absolu qu'on croit et donc que le hasard local n'est peut-être que l'aboutissement indiscernable d'une nécessité de plus grande envergure. Peut-être au demeurant dit-il aussi, à la Zénon, qu'aucune rencontre n'a jamais vraiment lieu et qu'un texte, par exemple, n'est jamais que la mise en mots d'un leurre, l'ombre d'une proie sans cesse enfuie et sans cesse poursuivie.

\section{LA RENCONTRE ET L'HABITUDE}

Au départ, donc, se trouvent mises en jeu et contredites l'une par l'autre la familiarité, l'habitus ${ }^{11}$ que suppose tout dialogue et la rencontre inattendue, espérée (comme la réponse que la religieuse attend à sa lettre) ou encore plus problématique, effacée, comme déniée, de se perdre dans la nuit des temps de la fiction virtuellement antécédentée, comme c’est le cas à la fois de Jacques ("Comment s'étaient-ils rencontrés? Par hasard, comme tout le monde") et du Neveu de Rameau sur la lecture duquel nous assurerons notre arpentage de la non-coïncidence incipitale.

Le Neveu s'ouvre justement sur une habitude qui s'énonce souveraine au point de nier, non pas le temps, comme il lui siérait, mais la température: "Qu'il fasse beau, qu'il fasse laid, c'est mon habitude d'aller sur les cinq heures du soir me promener au Palais-Royal» ${ }^{12}$. Les conditions atmosphériques dont l'incidence sur le mode de vie du narrateur est dite absolument nulle, du moins en ce début, sont une façon d'escamoter un emploi du temps réduit à la régularité d'une heure et d'une alternative sans effet. À l'intérieur de cette bulle temporelle qui relève plus du temps du mythe que de celui d'une quelconque socialité, le je est souverain, absolument. Pour les autres, il fait partie du paysage et de sa permanence: "C'est moi qu'on voit toujours seul, rêvant sur le banc d'Argenson» poursuit le texte. Quant à lui, il se suffit parfaitement à lui-même: "Je m'entretiens avec moimême de politique, d'amour, de goût ou de philosophie». Mieux, ce sujet que les circonstances extérieures ne sauraient faire dévier de sa trajectoire, une fois retranché, à son heure, à l'intérieur de luimême, et se tenant complaisamment lieu d'interlocuteur, se livre à tous vents:

J'abandonne mon esprit à tout son libertinage. Je le laisse maître de suivre la première idée sage ou folle qui se présente, comme on voit, dans l'allée de Foy, nos jeunes dissolus marcher sur les pas d'une courtisane à l'air éventé, au visage riant, à l'œil vif, au nez retroussé, quitter celle-ci pour une autre, les attaquant toutes et ne s'attachant à aucune. Mes pensées, ce sont mes catins. (Ibid.)

On voit d'ici le tableau: le philosophe au jardin est lui-même un jardin où cela circule et se poursuit dans une énergétique mentale qui est aussi une érotique fondée sur l'intermittence et l'évanescence de la séduction. Pour un peu l'on se croirait dans un paysage de Lucrèce où se déclinent des atomes en un 
incessant mouvement. Ce qu'il a sous les yeux est l'image de ce qu'il a dans la tête. Façon presque explicite d'annoncer à l'avance que Jean-François Rameau c'est lui, une fantasmagorie projetée à l'extérieur depuis la caverne de sa tête. Et ce «moi» qui n'était encore qu'une image extérieure, «sur le banc d'Argenson", au bout du regard de quiconque, va devenir bientôt le contrechamp et même le contrechant de «lui». Car Le Neveu de Rameau c'est aussi l'histoire de l'émergence progressive d'une parole théâtralisée, celle de Jean-François, ondoyante, multiple, contradictoire, séductrice (comme les «catins» de pensées du narrateur) et devant cette parole, l'autre, l'initiale, celle du narrateur devenu commentateur, se fait ancillaire, retenue et comme surgissant du trou du souffleur; elle sert de faire-valoir, de décor même. La voix dans la tête s'est projetée à l'extérieur, non pas côté jardin, sur ce lieu de l'Autre anarchique qu'aurait pu lui être le commerce érotique des jeunes gens mais, côté café, sur l'espace réglé d'une scène où l'on risque des coups qui peuvent aussi bien être de gueule et où le blanc et le noir, y compris dans leur connotation morale, visiblement s'échangent. Car l'incipit se fonde sur une incontestable symétrie: l'homme seul sur son banc dont les jeux intellectuels ressemblent aux jeux érotiques qu'il a sous les yeux donne lieu à la rencontre de deux hommes, qui sont plutôt deux instances, et qui commercent à l'enseigne du jeu d'échecs. Si les pensées du philosophe, à l'extérieur, étaient d'aimables catins (le mot «courtisane» appliqué aux jeunes filles qu'il a sous les yeux nous rappelle que «catin» n'a pas ici le seul sens de "poupée»), la discussion qu'il mène, à l'intérieur, avec un autre "dissolu» de l'espèce de ceux qu'il voyait tantôt muser au jardin, est ponctuée de coups, et plus précisément encore de gambits puisque tout ou presque y est affaire de provocations soigneusement calculées.

Mais comment s'est effectuée la rencontre, c'est-àdire, somme toute, l'émergence de la voix dialogique, son épiphanie? Comment l'incessant murmure dans la tête a-t-il pu «sortir» au point de se multiplier et de brûler les planches, sous les traits distordus d'un fantoche bien pratique? Comment cet anonymat d'ouverture, que nous appellerons par commodité «Diderot», devient-il ce pseudonyme au nom emprunté, mais familial, ou plutôt emprunté parce que familial, de «Rameau»? Comment devient-on Denis Rameau ou Jean-François Diderot et toutes les combinaisons là entre?

En rentrant, tout simplement. Au café, c'est-à-dire au lieu de la socialité où le solitaire extérieur du début du texte, celui «qu'on voit», maintenant à son tour s' "amuse à voir jouer aux échecs» (ibid.). Un «on" implicite ("on» joue aux échecs dit en fait la formule impersonnelle, et «je» les regarde) nous fait mieux percevoir ce qui, dans le regard, a changé. Le «on» du début du texte se répartissait en deux occurrences qui avaient toutes deux le même poids: l'affirmation de ce que j'appellerais le regard des circonstances, sorte de point focal qui, à force de pouvoir être occupé par quiconque, n'est plus que le parti pris des choses sans les hommes ${ }^{13}$, du moins tel que seuls les hommes peuvent l'imaginer et les circonstances, justement, le commander. En effet, le «on" de "c'est moi qu'on voit» désigne quiconque se trouve là quand j'y suis, quiconque, donc, est «solidaire» de moi, au sens étymologique, c'est-à-dire quiconque partage le même espace. Mais ce "quiconque» est une abstraction, une absence que seule la présence du moi rend possible, car c'est par une projection du moi jusqu'à ses limites extrêmes, le regard de l'autre, que, tel un halo, elle se forme. Le «on" du «comme on voit, dans l'allée de Foy, nos jeunes dissolus» n'a pas tout à fait la même portée. Du simple fait qu'il peut, cette fois, inclure le «je» et l'efface donc en tant qu'objet pour l'inclure dans le regard universel potentiel. Autrement dit, le saut dans la métaphore (et depuis la métonymie, car le «je» d'une certaine façon est là, il est, dans la fiction de sa présence, contigu à ces jeunes gens) est un saut dans le regard de l'Autre. Et c'est, dès lors, lorsque contemplant les joueurs d'échecs, le «je» assumera tout entier le regard de l'Autre qui voit qu' «on" joue, que l'Autre enfin pourra lui sauter aux yeux mais comme une métaphore secrète de lui-même, surgie comme par l'effet de cette spécularité virulente: 
Un après-dîner, j'étais là, regardant beaucoup, parlant peu et écoutant le moins que je pouvais, lorsque je fus abordé par un des plus bizarres personnages de ce pays où Dieu n'en a pas laissé manquer. (Ibid., p. 396)

Le dialogue va pouvoir prendre place, comme une sorte de ventriloquisme dans le miroir, et même comme si le miroir parlait. À qui s'est presque retranché de toute parole, dite ou entendue, pour n'être presque que regard, advient, superlative, la rencontre du porteur de parole, comme une pentecôte hallucinée.

Mais la théâtralité de la surenchère ( «un des plus bizarres» d'un pays qui n'en manque pas) joue moins l'événement que, au contraire, l'habitus, le lieu commun. Car ainsi introduit, Jean-François sera, au fond, exemple plus qu'exception et ce qui pourrait passer pour sa singularité la plus absolue n'est que le signe de son appartenance; s'il y a événement ce n'est que celui de la ponctualité d'un token, pour parler peircien. La rencontre n'est qu'une coupe, le bizarre un isolat. Comme l'était déjà, à son niveau, cet exemplaire «un après-dîner» dont la stratégie incipitale dit assez qu'il ne saurait être pris pour un banal «il était une fois». En effet, d'une part, on le sait, «l'aprèsdîner» correspond fort bien, au XVIII siècle, au «sur les cinq heures» d'ouverture, d'autre part et surtout, l'élection de ce jour particulier répond à une tactique de l'exception rigoureusement inverse de celle qui s'attache au personnage du neveu: celui-ci était une exception parmi les exceptions, celle-là est une exception parmi d'autres exceptions, certes, mais des exceptions qui ont ceci de particulier qu'elles se marquent comme une sorte de supplément (une exception de l'exception?) par rapport à l'alternative, pourtant exclusive, entre le beau et le mauvais temps. De deux choses, non pas l'une mais l'exceptionnel de l'une dit le texte. Car si, on s'en souviendra, le narrateur s'affirmait d'abord indifférent aux conditions atmosphériques, c'était pour ajouter, à peine quelques lignes plus bas, un fort surprenant: « $\mathrm{Si}$ le temps est trop froid, ou trop pluvieux, je me réfugie au café de la Régence». Le «trop» du climat représente donc à la fois la limite de la toute-puissance du narrateur ou ce qui excède sa capacité d'indifférence aux intempéries et le point de départ d'une autre habitude, présentée ainsi comme une alternative et effaçant littéralement rétrospectivement le «qu'il fasse laid» de la première phrase. Quand (et non si) il fait trop laid, c'est entendu, il se réfugie au café. Ainsi le temps superlatif (plus fort que toute alternative, comme si se soulignait ainsi l'indépassable ponctualité de l'instant, c'est-à-dire de l'aléatoire) force-t-il mais habituellement (une habitude contraire à la première, donc) le narrateur à rentrer voir les joueurs d'échecs et à finalement se faire aborder par l'original superlatif.

Encore faut-il remarquer qu'un relais s'est avéré nécessaire: entre les intempéries et l'intempestif, entre le climat (de ce pays, bien entendu) et Jean-François Rameau, original parmi les originaux que sont les habitants de ce pays, officient les joueurs d'échecs de ce café, crème de la crème, superlatifs parmi les superlatifs (de cette ville): «Paris est l'endroit du monde, et le café de la Régence est l'endroit de Paris, où l'on joue le mieux à ce jeu». Du «moi» au «lui», on le voit, s'opère un discernement progressif, un départage d'avec la masse ou le fond desquels émerge toujours quelque exception exemplaire mais bientôt rentrée dans le rang de son type, pour parler encore percien. C'est ainsi, par clivages successifs et littéralement par une «théorie des exceptions» que «moi» finit par rencontrer «lui» et que le «je» se frotte à l'Autre. Ainsi le personnage du neveu naitt-il visiblement du texte, comme une stase dans sa dérive incessante plutôt que comme un instant dans ce qui serait son explicitation du commencement. Et que Jean-François Rameau ait effectivement existé, que Diderot l'ait assurément connu, que le neveu du grand Rameau ait peut-être ressemblé à cela, ne change rien à l'affaire. Car ce qui se dit à travers lui c'est comment, à partir du familier anonyme (l'auteur, le lecteur en tant qu'entités abstraites présentes d'entrée de jeu), le texte fait de l'Autre et lui donne un nom: "Call me Ishmaël»" dira génialement ${ }^{14}$ Melville, moins d'un siècle plus tard. Ainsi le seul début que connaisse jamais un texte est-il celui, arbitraire et ironique, qui prend la forme d'un baptême mais dérisoire et au fond sans 
importance. Car comme le dit le narrateur de Beckett, on ne fait jamais, toujours, que continuer.

\section{LA LIMITE INDISCERNABLE}

En tant que sujet, nul ne sait jamais où commence ni où finit son espace. Pas plus qu'il ne saurait dire à partir d'où l'Autre règne, sous les espèces combinées d'autrui et du monde, ou ce que Lacan désigne comme l'ensemble des signifiants en tant qu'ils sont perçus comme formant un tout. Né d'un rapport fondateur (mais uchronique) à ce lieu de l'Autre, et toujours à renégocier, l'espace du sujet est fait de brèches et de débordements. Et nul ne saurait non plus dire quand il a commencé et quand il finira. Ces événements, comme ces limites, nous sont à jamais inaccessibles.

Mais ils et elles forment une structure. Une structure que l'espace littéraire, dans son balisage de l'imaginaire et du ponctuel, inverse pour mieux la dire; en effet, tous les débuts sont rétrospectifs, c'est une des plus anciennes leçons de la littérature. Un texte ne part jamais que d'une ponctualité suspendue, ne serait-ce que le temps de la dire, et tout incipit se donne ainsi pour une commotion, une déflagration, une sorte de Big Bang en deux dimensions: vers la suite possible et vers une antériorité à la fois toujours improbable et toujours supposée.

Mais ce qui surtout se met en scène dans tout incipit, c'est l'occupation opiniâtre, l'arpentage obstiné du présent, insaisissable et inévitable, de l'écriture. Qui se donne à partager. Comme le décentrement de la séduction, une réponse à l'appel de l'Autre, une esquive amoureuse en forme d'esquisse toujours en cours d'inaccomplissement.

\section{N O TES}

1. L.- F. Céline, Voyage au bout de la nuit, Paris, Gallimard, coll. "Folio", 1997, p. 7.

2. Ibid., p. 505.

3. A. Robbe-Grillet, Dans le labyrinthe, Paris, Minuit, 1959, p. 220-221

4. À cet égard on remarquera la même dimension métalangagière implicite que celle qui dans le futur du projet initial (implicitement une sorte de « je vais donc vous raconter comment ça a débuté») organisait la séparation de la voix entre narrateur et personnage; il s'agit ici, comme il se doit, de les résorber l'une dans l'autre mais visiblement dans le "on" universel (non seulement "nous ici maintenant", qu'il s'agisse des personnages ou du rapport «auteur "-« lecteur » mais " qui que ce soit").

5. A. Robbe-Grillet, Le Voyeur, Paris, Minuit, 1955, p. 9.
6. Du moins en droit car il va de soi que rien de la fiction n'a lieu avant la lecture qui l'actualise.

7. D. Diderot, La Religieuse, dans Euvres romanesques, Paris, Éd. Garnier frères, 1959, p. 235.

8. On se souviendra en effet que le roman naquit d'une farce faite au marquis et qu'avant de se laisser prendre lui-même au jeu, au point, si l'on en croit Grimm de pleurer aux malheurs de son imaginaire cloîtrée, Diderot, jouant la religieuse malgré elle, écrivit des lettres suppliant M. de Croismare de venir à son aide. L'incipit évoque donc ici des réponses qui vinrent réellement, hors fiction si l'on peut dire en l'occurrence.

9. Suit en effet cette phrase d'ouverture une description des qualités de M. de Croismare qui se termine par la première indication, très discrète, un simple participe passé accordé au féminin. quant au sexe du personnage: «Et j'ai jugé par le vif intérêt qu'il a pris à mon affaire et par tout ce qu'on m'en a dit que je ne m'étais point compromise en m'adressant à lui " (ibid.). À ce stade, le lecteur ne sait encore rien de l'«affaire" du personnage et pour peu qu'il soit un peu distrait et n'ait pas remarqué le participe passé, il lui faudra encore quelques lignes pour savoir hors de tout doute que c'est une femme qui parle, celle-ci se présentant d'abord par son rapport à ses «sœurs", son destin s'activant ironiquement du jeu de mots qui la fera devenir «sœur», tout comme, plus tard, le "frère" de Jacques deviendra "frère ". Cette prise au mot est en fait une dénonciation de l'artificialité de l'appellation religieuse. Pour Diderot, la nature est du côté de la famille et il voue, on le sait, au cloître - y compris pour les raisons biographiques qui le font aussi jouer ici les «sœurs " pour venger sa sœur Angélique morte folle au couvent - une exécration qui ne se démentira jamais.

10. En ce sens, le lecteur, derrière M. de Croismare, occupe ici la place de la déesse, à l'ouverture de l'Iliade: «Chante, déesse, la colère d'Achille» dit en effet plutôt "permets, déesse, que je chante». Et l'anonymat historique d'Homère fait fort à point de cette déesse quelque chose comme la littérature.

11. Au sens, certes, de lieu commun partagé, comme il s'entend généralement en sociologie, mais aussi de lieu commun solipsiste, si l'on me permet l'oxymore, dans la mesure où ce lieu commun «individuel» représente pour le sujet une immersion confortable dans un espace et un temps autres, déjà balisés, le lieu par excellence du non-événement, le lieu où le sujet n'a pas besoin de se revendiquer, assuré qu'il est par toute la force anonyme que lui donne l'espace de l'habitude.

12. D. Diderot, Le Neveu de Rameau, dans Euvres romanesques, op. cit., p. 395

13. Lacan a magistralement analysé le dispositif que je tente de mettre à jour ici, dans «la ligne et la lumière» (Le Séminaire, Livre XI. Les Quatre Concepts fondamentaux de la psychanalyse, Paris, Éd. du Seuil, p. 85-96), notamment en racontant l'histoire de ce marin, Petit-Jean, qui un jour, désignant un objet flottant à la surface de la mer lui dit, à la blague: "Tu vois, cette boîte? Tu la vois? Eh bien, elle, elle te voit pas!". Commentaire de Lacan: "Ce petit épisode, il trouvait ça très drôle, moi, moins. J'ai cherché pourquoi moi je le trouvais moins drôle. C'est fort instructif. D'abord, si ça a un sens que Petit-Jean me dise que la boîte ne me voit pas, c'est parce que, en un certain sens, tout de même, elle me regarde. Elle me regarde au niveau du point lumineux, où est tout ce qui me regarde, et ce n'est point là métaphore». Et plus loin: "Je ne suis pas simplement cet être punctiforme qui se repère au point géométral d'où est saisie la perspective. Sans doute, au fond de mon œil, se peint le tableau. Le tableau, certes, est dans mon œil. Mais moi, je suis dans le tableau " (p.89).

14. Génialement parce que dans un contexte biblique, Ismaël est le nom de l'Autre par excellence, celui que l'on repousse au désert. Et, aussi bien, le premier métèque de l'histoire. 